\title{
Solid-state proton NMR of paramagnetic metal complexes: DANTE spin echoes for selective excitation in inhomogeneously broadened lines
}

\author{
Diego Carnevale ${ }^{\mathrm{a}, *}$, A.J. Perez Linde ${ }^{\mathrm{a}}$, Gerald Bauer ${ }^{\mathrm{a}}$, Geoffrey Bodenhausen ${ }^{\mathrm{a}, \mathrm{b}, \mathrm{c}}$ \\ a Institut des sciences et ingénierie chimiques (ISIC), Ecole Polytechnique Fédérale de Lausanne (EPFL), 1015 Lausanne, Switzerland \\ ${ }^{\mathrm{b}}$ Département de Chimie, Ecole Normale Supérieure, 75231 Paris Cedex 05, France \\ ${ }^{\mathrm{c}}$ UMR 7203, CNRS/UPMC/ENS, Paris, France
}

\section{A R T I C L E I N F O}

Article history:

Received 2 May 2013

In final form 25 June 2013

Available online 2 July 2013

\begin{abstract}
A B S T R A C T
The paramagnetic complex bis(oxazolinylphenyl)amine- $\mathrm{Fe}(\mathrm{III}) \mathrm{Cl}_{2}$ is investigated by means of solid-state proton NMR at $18.8 \mathrm{~T}(800 \mathrm{MHz})$ using magic-angle spinning at $65 \mathrm{kHz}$. Spin echoes that are excited and refocused by combs of rotor-synchronized pulses in the manner of 'Delays Alternating with Nutation for Tailored Excitation' (DANTE) allow one to characterize different chemical environments that severely overlap in conventional MAS spectra. Such sequences combine two apparently contradictory features: an overall bandwidth exceeding several $\mathrm{MHz}$, and very selective irradiation of a few $\mathrm{kHz}$ within inhomogeneously broadened sidebands. The experimental hyperfine interactions correlate well with DFT calculations.
\end{abstract}

(C) 2013 Elsevier B.V. All rights reserved.

\section{Introduction}

Paramagnetic species in solids often have such broad NMR spectra that they cannot be properly excited by a single pulse nor refocused to form spin echoes [1]. In recent years, several experimental approaches have been proposed to overcome these intrinsic difficulties both in static solids and under magic-angle spinning (MAS) conditions [2-4]. Investigations of systems containing paramagnetic centers have proved to be important for a variety of fields, ranging from the structure determination of metalloproteins [5-8] to the characterization of catalytic organometallic complexes [9-14]. We have recently shown that spin-echo techniques based on combs of rotor-synchronized pulses employing Delays Alternating with Nutation for Tailored Excitation (DANTE) [15-18] can be used to excite nuclei such as protons that experience severe inhomogeneous broadening due to nearby paramagnetic nuclei [19].

The excitation profile resulting from a comb of $N r f$ pulses of duration $\tau_{p}$ spaced by a delay $\tau$ features a series of narrow spikelets whose width is proportional to $\left(N \times\left(\tau+\tau_{p}\right)\right)^{-1}$, extending over a spectral width proportional to $\tau_{p}^{-1}$ [18]. When combined with magic-angle spinning solid-state NMR, DANTE excitation schemes can be applied in a rotor-synchronized fashion, i.e., with $N \times\left(\tau+\tau_{p}\right)=$ $\tau_{\text {rot }}$. When an inhomogeneously broadened powder pattern is broken up into spinning sidebands by the mechanical rotation, a proper choice of the carrier frequency allows one to uniformly excite a whole spinning sideband family over $\tau_{p}^{-1}$. This amounts to

\footnotetext{
* Corresponding author.

E-mail address: diego.carnevale@epfl.ch (D. Carnevale).
}

saying that the comb of excitation spikelets can be shifted in frequency domain to match the spinning sideband family of a nuclear species in a given environment. We have shown [19] that if one applies only one pulse of length $\tau_{p}$ per rotor period $(N=1)$ and if the DANTE burst extends over $K$ rotor periods, any cumulative flip angle can be achieved by virtue of the synergy between the $r f$ pulses and the rotational echoes, as long as homogeneous decay in the pulse intervals can be neglected. We have indicated this scheme by the symbol $D_{1}^{K}$. We have shown [19] that DANTE schemes can achieve not only broadband excitation, but also broadband refocusing and broadband inversion, or indeed any arbitrary flip angle, covering spectral widths as large as several $\mathrm{MHz}$ at $\mathrm{rf}$ field strengths as low as $30 \mathrm{kHz}$.

In this Letter, we use very fast spinning rates near $v_{\text {rot }}=65 \mathrm{kHz}$ and investigate inhomogeneous broadening of proton resonances in the paramagnetic complex bis(oxazolinylphenyl)amine$\mathrm{Fe}(\mathrm{III}) \mathrm{Cl}_{2}$ that may be relevant for stereoselective catalysis. Chiral derivatives of this complex were successfully employed in hydrosilylation reactions of aromatic ketones [20-22]. We show that by using a large number of rotor periods $K$, the width of the $r f$ spikelets can be made so narrow that one can tune the selectivity of the DANTE scheme to monitor barely resolved and partly overlapping signals stemming from inhomogeneous environments.

One can cover the full lineshape by moving the carrier frequency across a spectral range equal to the spinning frequency. This permits one to obtain site-specific information normally hidden by inhomogeneous broadening. To the best of our knowledge, there is no other technique in solid-state NMR that allows such a selective exploration. 


\section{Results and discussion}

The structure of the Fe(III) metal complex considered in this Letter is shown in Figure 1a. Three structures optimized with DFT methods for three possible spin states $S=1 / 2,3 / 2$ and $5 / 2$ are overlaid in Figure $2 \mathrm{~b}$. For such compounds, a simple pulse-acquire sequence results in severely distorted lineshapes which are unsuitable for interpretation [23]. This is due to dephasing occurring during the dead time before acquisition that cannot be properly treated by a phase correction of the resulting spectrum as well as to a non-uniform excitation of the entire spectral width. A more efficient approach which overcomes the former problem consists in utilizing spin echo experiments [24,25], allowing the signal acquisition to be triggered at the top of a rotational echo where inhomogeneous dephasing is refocused. Only spin-echo experiments have been considered in this Letter. The desired $p=0 \rightarrow p=+1 \rightarrow p=-1$ coherence-transfer pathway [26] can be selected either by using conventional rectangular $90^{\circ}$ and $180^{\circ}$ pulses or with DANTE combs $D_{1}^{K}-\tau-D_{1}^{2 K}-\tau$ of rotor-synchronized pulses. Figure 2a shows a spin-echo spectrum of the compound shown in Figure 1. Inhomogeneous broadening due to the paramagnetic center is clearly visible. However, it is debatable whether the full anisotropic hyperfine spinning-sideband pattern has been uniformly excited. Figure $2 b$ shows an expansion of a spectral region which matches the spinning frequency $v_{\text {rot }}=65$ $\mathrm{kHz}$, extending from the centerband at ca. $12 \mathrm{ppm}$ to the first spinning sideband to its right (i.e., towards lower frequency). A second component can be clearly identified at ca. -15 ppm. However, a fit of this spectrum assuming two sites with different CSA tensors (or, equivalently, different hyperfine coupling tensors) results in a very poor agreement with the experimental data. This is presumably due to the presence of (at least) a third site that gives rise to a spinning-sideband family that is completely hidden underneath the two main components because of its much larger anisotropy. An attempt to fit with three different sites however failed to reproduce the experimental spectrum. This is probably due to a nonuniform excitation across the whole spectrum as well as to homogeneous broadening which results in severe overlap. The selectivity of the $r f$ spikelets resulting from DANTE combs allows one to probe a specific environment present in the sample. By stepping the $r f$ carrier frequency across the interval between two spinning sidebands, it is possible to extract both isotropic and anisotropic hyperfine information contained in the whole lineshape of Figure 2a. The choice of $N$ and $K$ for DANTE spin-echoes is important for such purposes since the selectivity of the comb can be tuned at will through these two parameters. For the measurement of inhomogeneous broadening, $N=1$ allows the excitation of all spinning sidebands belonging to a given site. A reasonable flip angle is then achieved by repeating the DANTE unit $K$ times for excitation and $2 K$ times for refocusing. In order to enhance site-selectivity, one can increase $K$ so to reduce the width of the $r f$ spikelets. Signal (a)

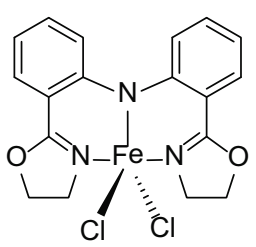

(b)

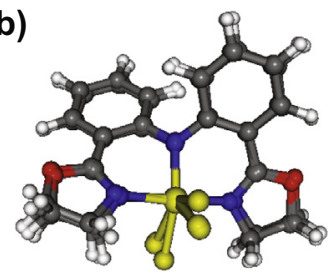

Figure 1. (a) Structure of the paramagnetic complex bis(oxazolinylphenyl)amine$\mathrm{Fe}(\mathrm{III}) \mathrm{Cl}_{2}$ investigated in this Letter by solid-state proton NMR. (b) Overlay of three DFT-optimized structures of the Fe complex for low-, intermediate- and high-spin states $S=1 / 2,3 / 2$ and $5 / 2$ optimized with the hybrid functional B3LYP, LANL2DZ and $6-31+G(d)$ basis sets for Fe and all other nuclei, respectively. losses due to homogeneous decay, on the other hand, can be minimized by decreasing $K$ and increasing the pulse length $\tau_{p}$.

In our sample, we found a good compromise with $N=1$ and $K=5$. Such DANTE experiments can be referred to as $D_{1}^{5}-\tau-D_{1}^{10}-\tau$. Given an $r f$ field strength $v_{1}=250 \mathrm{kHz}$ and using a pulse length $\tau_{\text {rot }}=0.2 \mu \mathrm{s}$, our DANTE excitation scheme corresponds to a $90^{\circ}-\tau-180^{\circ}-\tau$ spin echo. At the spinning rate $v_{\text {rot }}=65-$ $\mathrm{kHz}$ used in this Letter (rotor period $\tau_{\text {rot }}=1 / v_{\text {rot }}=15.38 \times 10^{-6} \mathrm{~s}$ ) such combs of pulses last 17 rotor periods and produce spikelets in the $r f$ excitation spectrum spaced at multiples of $v_{\text {rot }}=65 \mathrm{kHz}$, each with a width of about $\left(17 \times 15.38 \times 10^{-6} \mathrm{~s}\right)^{-1}=3.8 \mathrm{kHz}$. This is much narrower than the inhomogeneous breadth of the spinning sidebands, so that the net effect is to excite a narrow 'pencil' within the breadth of each sideband. Figure $2 \mathrm{c}$ and $\mathrm{d}$ show the anisotropy $\Delta_{\text {aniso }}$ and asymmetry $\eta$, respectively, obtained from fits of $D_{1}^{5}-\tau-D_{1}^{10}-\tau$ spin-echo spectra performed while shifting the carrier frequency across the spectral window shown in Figure $2 \mathrm{~b}$ (equal to the spinning frequency) in 18 steps of $4 \mathrm{kHz}$. The points shown in Figure $2 \mathrm{c}$ and $\mathrm{d}$ represent the anisotropy and asymmetry parameters obtained by fitting spectra acquired with a carrier frequency set to a position stepped across the spectral window of Figure $2 \mathrm{~b}$. In such a manner one can selectively monitor, in 'slices' of a breadth of only $3.8 \mathrm{kHz}$, the distribution of chemically distinct sites in samples experiencing different anisotropic hyperfine interactions across the inhomogeneously broadened lineshape. The fits have been carried out assuming a single site. The variation of the anisotropy $\Delta_{\text {aniso }}$ across the spectral width explored shows however that at least three different environments must be present. Starting at ca. $12 \mathrm{ppm}$, where we found a site A with $\Delta_{\text {aniso }}=150$ $\mathrm{ppm}$, the $r f$ carrier is progressively shifted to a second environment B (identifiable also in Figure $2 \mathrm{~b}$ at ca. $-15 \mathrm{ppm}$ ) characterized by a $\Delta_{\text {aniso }}=200 \mathrm{ppm}$. At ca. $-38 \mathrm{ppm}$ a third environment $\mathrm{C}$ has a much larger anisotropy $\Delta_{\text {aniso }}=400 \mathrm{ppm}$. In contrast to the previous two, the broad signal of the environment $\mathrm{C}$ is completely hidden under the envelope of spinning sidebands of the two sites $A$ and $B$ in Figure $2 a$ and $b$. The isotropic shifts of the three sites as $A, B$ and $C$ are indicated by red arrows in Figure $2 b$ and $c$. The DANTE echo method allows one to unveil structural information that is completely invisible in conventional MAS spectra. Figure $2 \mathrm{~d}$ shows the distribution of the asymmetry parameter $\eta$ over the spectral range considered. The asymmetry is more uniform than the anisotropy. The determination of $\eta$ by MAS NMR is known to be more prone to errors than the anisotropy $\Delta_{\text {aniso }}$ [27]. It is worth stressing at this point that the parameters extracted by our single-site fits are merely meant to identify variations across the spectral range considered, rather than to determine the anisotropic hyperfine interactions of individual sites. In fact, more than one site needs to be considered simultaneously to reproduce the observed lineshapes. Nonetheless, the variations identified by single-site fits allow one to estimate the number of distinguishable environments in the sample. In our Fe(III) complex, the above analysis of the distribution of anisotropic hyperfine parameters across the lineshape suggests that one can distinguish (at least) three different sites A, B and C with isotropic shifts at ca. 12, -15 and $-38 \mathrm{ppm}$, respectively. These shifts result from contributions of the isotropic parts of both the chemical shift and paramagnetic interactions.

Figure $3 \mathrm{a}-\mathrm{c}$ shows spectra acquired with DANTE echoes $D_{1}^{5}-\tau-D_{1}^{10}-\tau$ while the carrier frequency is centered on these three isotropic shifts. Fits calculated assuming only two sites are shown in red. A very good agreement is obtained with the experimental spectra.

We find that models assuming only two sites at the time can nicely describe the inhomogeneous broadening of our $D_{1}^{5}-\tau-D_{1}^{10}-\tau$ spectra. The inclusion of a third site does not sig- 
(a)

(b)
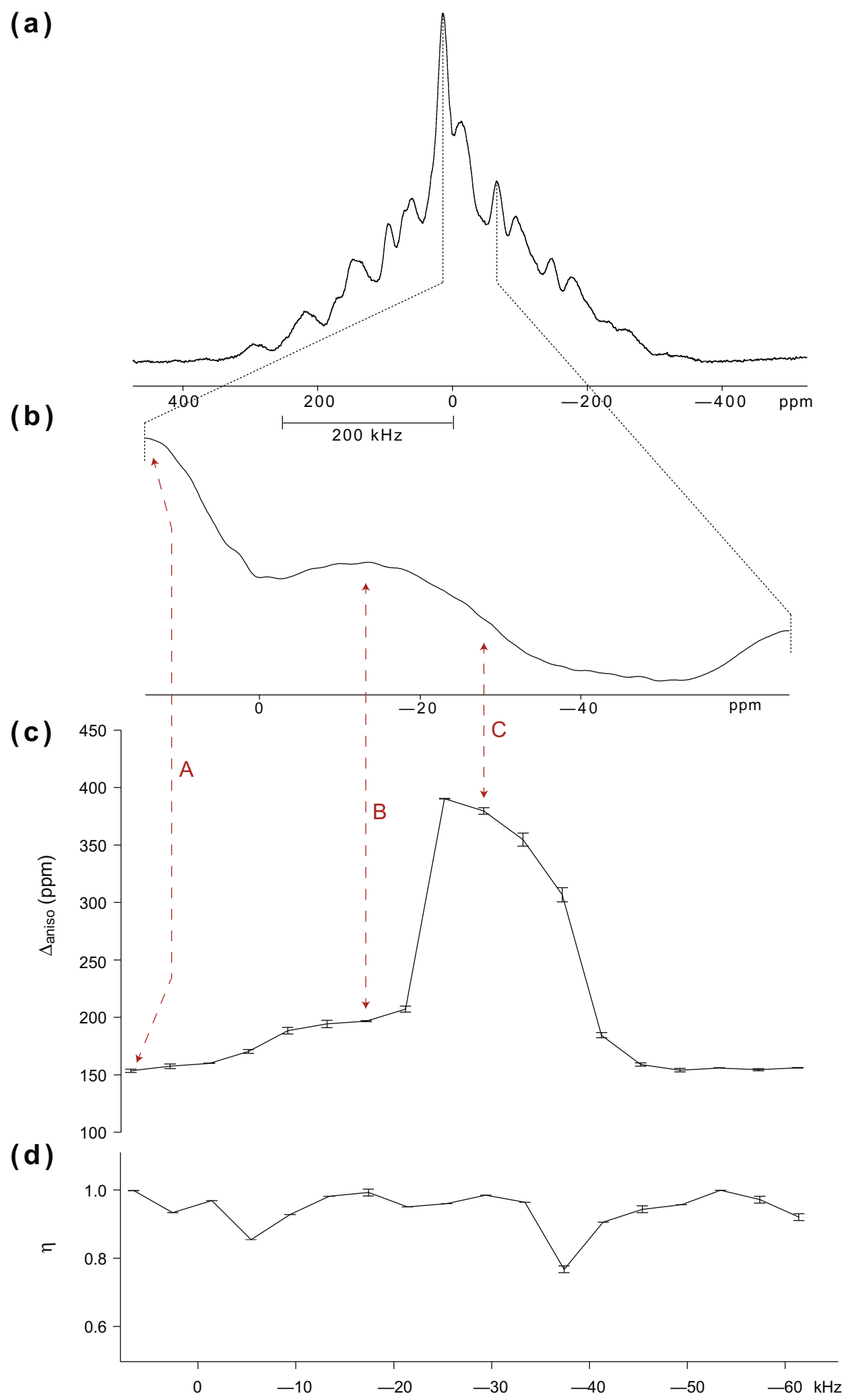

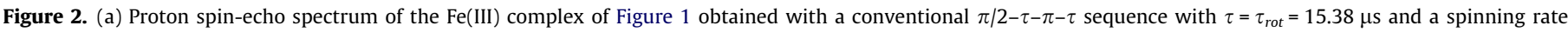

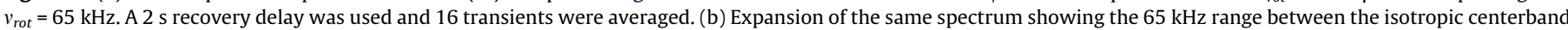

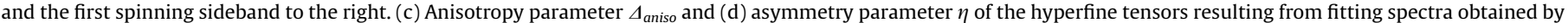

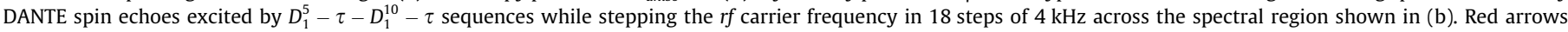
indicate three environments $\mathrm{A}, \mathrm{B}$ and $\mathrm{C}$ that can be distinguished because of marked variations of the anisotropy parameter $\Delta_{\text {aniso. }}$ 
(a)

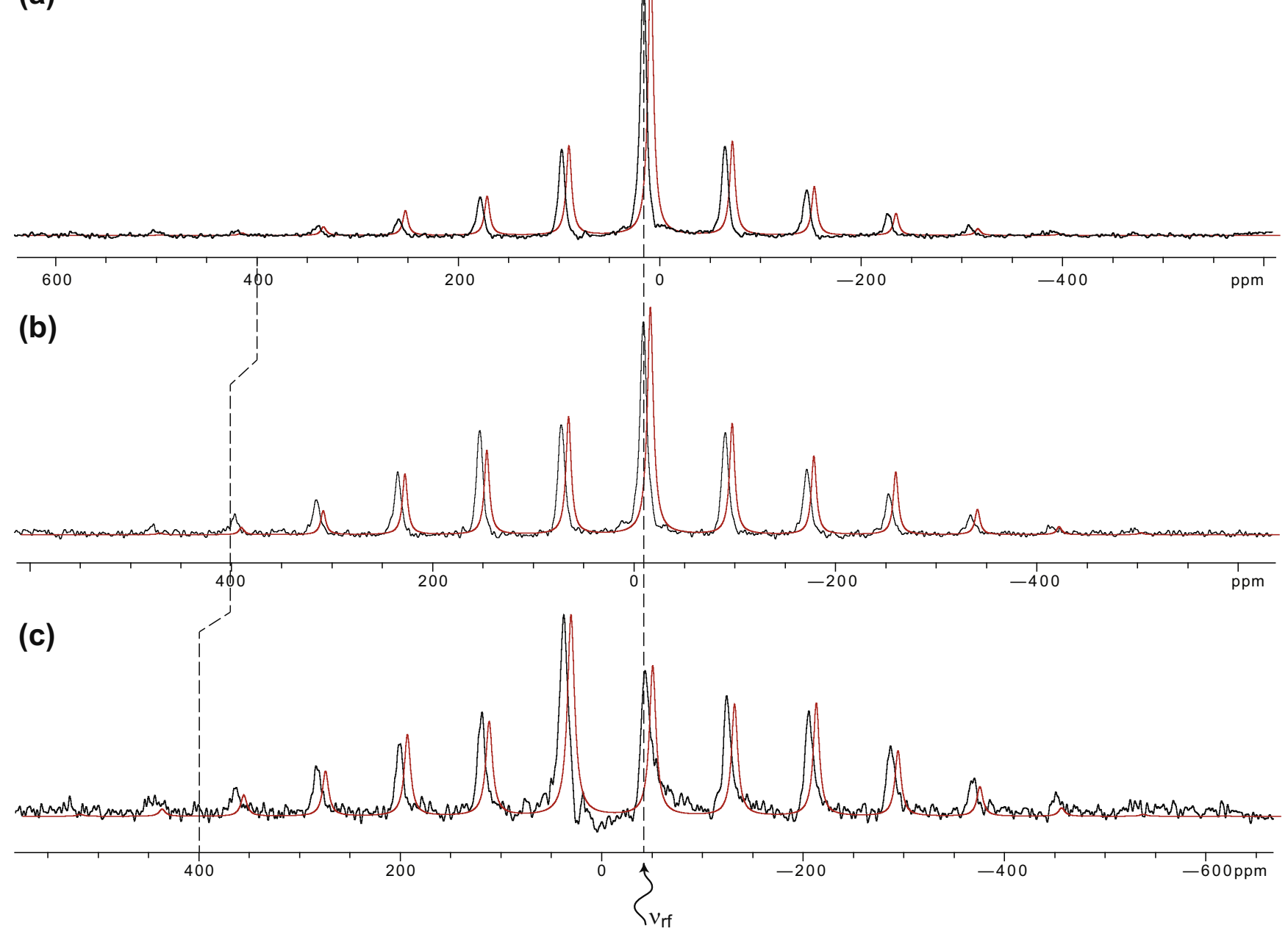

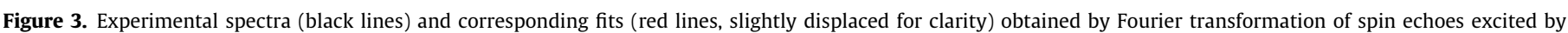

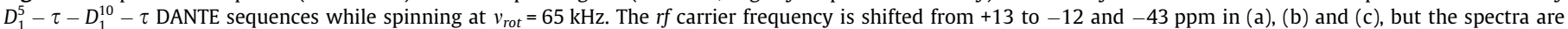

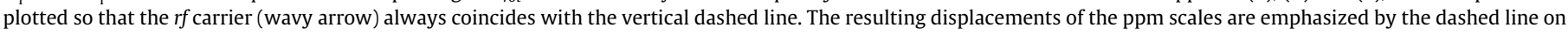

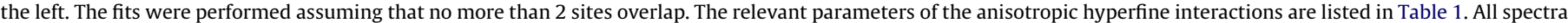
resulted from averaging of 64 scans, with a recovery delay of $2 \mathrm{~s}$.

nificantly improve the quality of the fits. This is due to the fact that the two environments A and B experience a very similar inhomogeneous broadening and can thus be reasonably well merged into a single site. On the other hand, site $\mathrm{C}$, which is completely hidden when observing conventional echoes, needs to be considered in the fits, since it is responsible for the largest anisotropic hyperfine contributions to the spectra.

The parameters of the two sites considered in the fits of the spectra shown in Figure 3 are summarized in Table 1. It is worth noting that the largest anisotropic hyperfine interaction of site $C$ can now be more accurately estimated to $\Delta_{\text {aniso }}=443 \mathrm{ppm}$, while a crude single-site fit gave $\Delta_{\text {aniso }}=400 \mathrm{ppm}$. Sites A and B were re- fined to have $\Delta_{\text {aniso }}=122$ and $92 \mathrm{ppm}$, respectively, while the crude single-site analysis lead to overestimate them to $\Delta_{\text {aniso }}=150$ and $200 \mathrm{ppm}$. The analysis of these data clearly shows that a single-site model underestimates the larger, and overestimates the smaller anisotropies. Once a two-site model is used, more reliable anisotropies are obtained, as testified by the quality of the fits shown in Figure 3.

The anisotropic part of the dipolar hyperfine coupling between a paramagnetic center and a nearby nuclear spin depends on their distance $R$ : [28-30]

$\left|\Delta_{\text {aniso }}\right|=2 c\left[\mu_{0} /\left(4 \pi R^{3}\right)\right]$,

Table 1

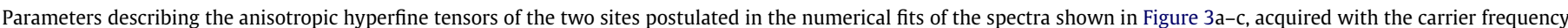

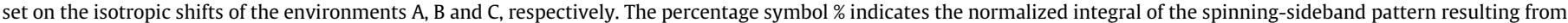
the corresponding site. The parameters which characterize the environments A, B and C are shown in bold. Site 2 always features the largest inhomogeneity.

\begin{tabular}{|c|c|c|c|c|c|c|c|c|}
\hline \multirow[t]{2}{*}{ Environment } & \multicolumn{4}{|l|}{ Site 1} & \multicolumn{4}{|l|}{ Site 2} \\
\hline & $\delta_{\text {iso }}(\mathrm{ppm})$ & $\Delta_{\text {aniso }}(\mathrm{ppm})$ & $\eta$ & $\%$ & $\delta_{\text {iso }}(\mathrm{ppm})$ & $\Delta_{\text {aniso }}(\mathrm{ppm})$ & $\eta$ & $\%$ \\
\hline A & 13.4 & 122.0 & 0.6 & 0.60 & 13.4 & 337.4 & 0.8 & 0.40 \\
\hline B & -12.0 & 91.9 & 0.7 & 0.24 & -11.8 & 355.7 & 1.0 & 0.76 \\
\hline C & 38.6 & 153.0 & 0.7 & 0.33 & -42.7 & 443.1 & 0.6 & 0.66 \\
\hline
\end{tabular}


where $c=g^{2} \mu_{B}^{2} S(S+1) /\left(3 k_{B} T\right), T$ is the temperature, $k_{B}$ is Boltzmann's constant, $\mu_{B}$ is the Bohr magneton, $g$ is the trace of the electron $g$-tensor and $S$ is the electron spin quantum number of the paramagnetic center. This latter constant is related to the number of unpaired electrons in the metal. The size of the anisotropy $\Delta_{\text {aniso }}$ can be conveniently expressed in units of ppm [30]:

$\left|\Delta_{\text {aniso }}\right|=\left[1.661 \times 10^{6} S(S+1)\right] /\left(R^{3} T\right)$,

where $R$ and $T$ are in units of $\AA$ and $K$, respectively. This model neglects intermolecular contributions to the anisotropic dipolar shifts. These contributions have been estimated in other studies [30] and are not considered here.

In order to check the accuracy of our measurements and the prospects of determining interatomic $\mathrm{H}-\mathrm{Fe}$ distances $R$ in our system, we have performed a computational investigation considering the three possible spin states $S=1 / 2,3 / 2$ and $5 / 2$. The structure of the molecule was first optimized with the semi-empirical method PM7 as implemented in MOPAC2012 [31]. A subsequent optimization was performed with Gaussian09 [32] using the B3LYP/6$31+G(d)$ level of theory. The effective-core potential LANL2DZ [33-35] basis set was used for the iron center. All these optimizations have been performed for each of the three possible spin states. The resulting global minima are shown overlaid in Figure $1 \mathrm{~b}$. The filled dots in Figure 4 show the anisotropies $\Delta_{\text {aniso }}$ that were predicted from the optimized structures as a function of the optimized H-Fe distance $R$, using Eq. (2) for $S=1 / 2,3 / 2$ and $5 / 2$. The distances calculated from the anisotropies $\Delta_{\text {aniso }}$ that were obtained from fits assuming two sites of the experimental spectra observed with DANTE echoes for $S=1 / 2,3 / 2$ and $5 / 2$ are instead shown with empty squares. The errors associated with the fitting process are smaller than ca. $\pm 2 \mathrm{ppm}$ and always fall within the size of the symbols of Figure 4. As discussed above, in a two-site model, one site takes into account the largest anisotropy of site $C$, and the other takes into account the other two sites A and B, both characterized by a smaller anisotropy. The two groups of empty squares associated with these sites are indicated in the figure for each spin state $S$. For the low-spin configuration with $S=1 / 2$ there is no agreement between the calculated and experimental data as there is an almost complete lack of overlap between the blue series of filled dots and empty squares. This allows one to exclude this electronic state. For the high-spin configuration $S=5 / 2$, the agreement is poor for the protons nearest to the paramagnetic metal, for which anisotropies $\Delta_{\text {aniso }}>1000 \mathrm{ppm}$ are predicted. The lack of experimental data for the nearest protons may be understood by recognizing that an anisotropic hyperfine pattern spreading over $1000 \mathrm{ppm}(0.8 \mathrm{MHz}$ at $18.8 \mathrm{~T})$ could not be excited uniformly because of the high quality factor of our probe. It is worth mentioning that the pulse length $\tau_{p}=0.2 \mu$ s used for our DANTE trains can ensure a uniform excitation over as much as $\tau_{p}^{-1}=5 \mathrm{MHz}$. If we assume that $S=5 / 2$, the experimental data also suggest distances as large as $R^{A, B}>7 \AA$ for the most remote protons, whereas our optimized structure predicts $R<6.6 \AA$ for these protons. The overlap between experimental and predicted values is best for $S=3 / 2$, for which all empty squares fall within the DFT-calculated range of distances. However, the experimental data slightly overestimate the distances for the closest environments at $R^{C}=3.5 \AA$. Also in this case, there is a poor agreement for the most remote protons $6.0<R<6.6 \AA$. In contrast with the $S=5 / 2$ case, these latter distances are instead underestimated to $R^{A, B}=5.5 \AA$.

These discrepancies may be attributed to the severe overlap of the sites that we could observe in our proton spectra. Only the two environments, A and B, can be readily identified in Figure 2a and $\mathrm{b}$. If $m$ is the number of crystallographically distinct molecules per unit cell, as many as $16 \times m$ proton resonances could be observed. Nonetheless, in the spin system under investigation, our method is capable of unveiling a third site $C$ with a much larger anisotropy. The method appears to be accurate, since the errors of the internuclear distances are limited to ca. $\pm 1 \AA$.

Because of the high isotopic abundance and large gyromagnetic ratios of protons, the homonuclear dipolar interactions are very large, so that structural NMR studies based on protons in solids

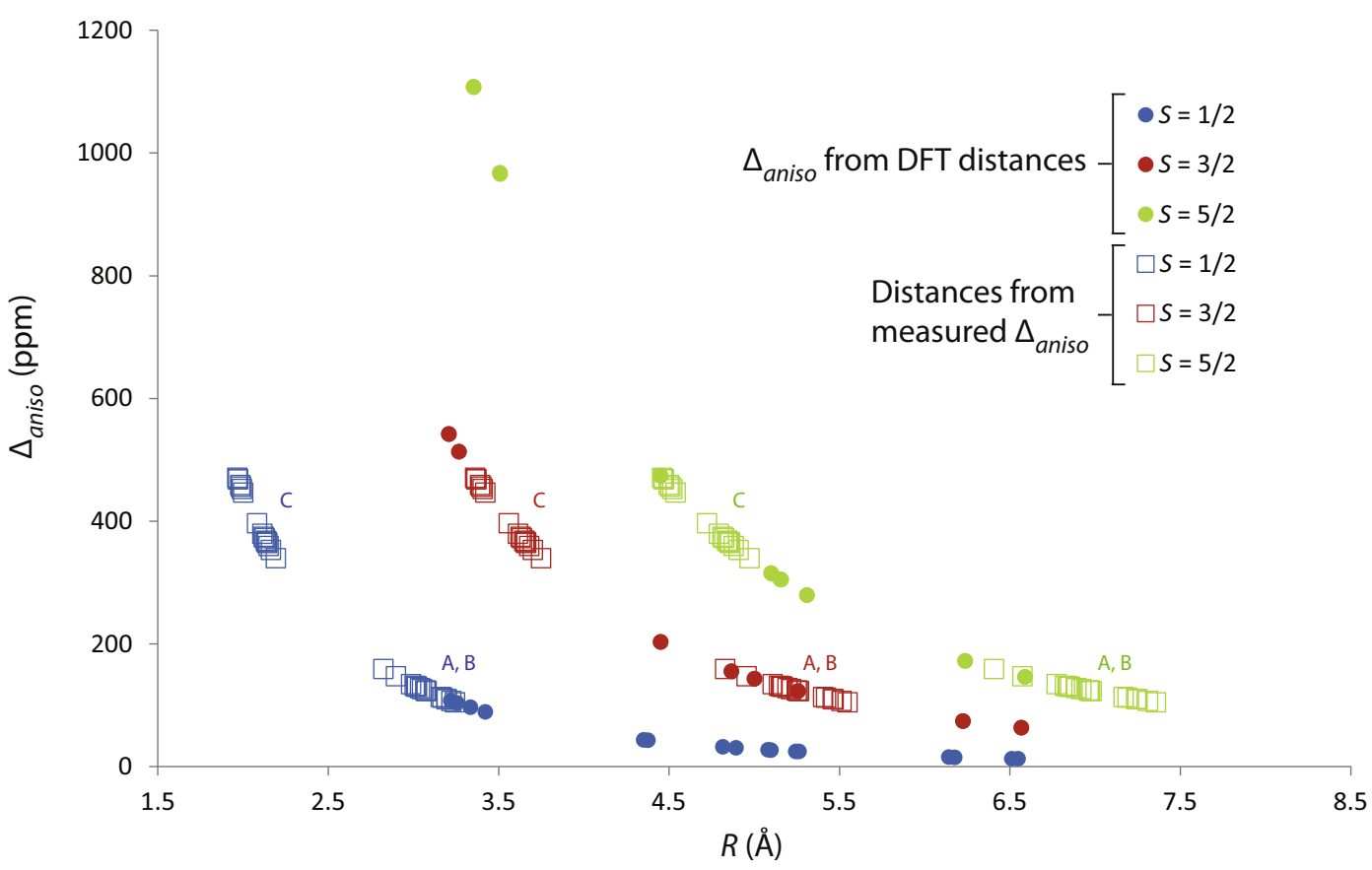

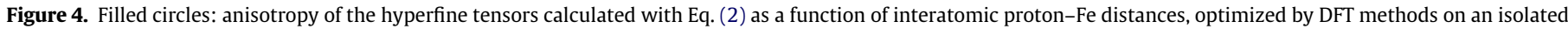

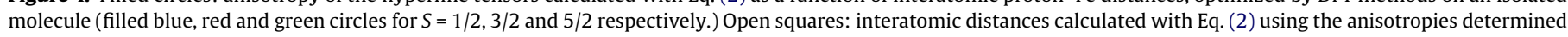

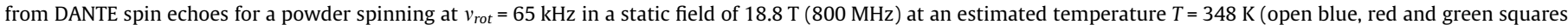
for $S=1 / 2,3 / 2$ and $5 / 2$, respectively). The errors for the latter series of data fall within the size of the squares. 
are challenging. In paramagnetic species, the presence of large inhomogeneous dipolar hyperfine couplings makes these systems amongst the most challenging that one can possibly deal with in NMR spectroscopy. Nonetheless, DANTE spin echoes have proven to selectively measure anisotropies under extremely demanding conditions.

For each of the three hypothetical electronic spin states $S=1 / 2$, $3 / 2$ and $5 / 2$, there are 16 filled dots in Figure 4 that correspond to the 16 protons in the metal complex bis(oxazolinylphenyl)amine$\mathrm{Fe}(\mathrm{III}) \mathrm{Cl}_{2}$. On the other hand, the 18 empty squares correspond to the 18 experiments performed over the spectral window of Figure $2 \mathrm{~b}$ by shifting the carrier frequency in 18 steps of $4 \mathrm{kHz}$. These 18 spectra sample the variations of the parameters that determine the inhomogeneously broadened spectrum of Figure 2a. Indeed we have been able to identify and fully characterize (see Table 1) three different environments A, B and C, the last of which is completely invisible in the spectral window of Figure $2 \mathrm{~b}$. These three environments can be thought of as three groups of protons which are found at three distinct (average) distances from the Fe(III) center. The optimized structures of Figure $1 \mathrm{~b}$ indicate that, for any of the three hypothetical electronic spin states $S=1 / 2,3 / 2$ and $5 / 2$, all 16 protons in the metal complex must be at a distance $3.2<R<6.6 \AA$ from the $\mathrm{Fe}(\mathrm{III})$ center. By 'binning' the 16 proton distances into three groups according to $R$ and subsequently taking the average distances within each group, one obtains three average distances derived from the structures that have been optimized by DFT calculations for the three spin states $S=1 / 2,3 / 2$ and $5 / 2$. These three groups can then be directly correlated with the three environments $\mathrm{A}, \mathrm{B}$ and $\mathrm{C}$. The resulting histogram is shown in Figure 5 a. The width of each box is $\left(R_{\max }-R_{\min }\right) / 3=(6.6-3.2) / 3=1.13 \AA$. The average distance within each box is represented by a vertical dashed line. For the three hypothetical electronic spin states $S=1 / 2,3 / 2$ and $5 / 2$ we used blue, red and green dashed lines, respectively. Figure $5 \mathrm{~b}$ shows correlations between the distances $R_{\exp }$ calculated from the parameters $\Delta_{\text {aniso }}$ that have been determined experimentally (see Figure 3 and Table 1 ) and the average distances $R_{\text {calc }}$ predicted from DFT-optimized structures. Clearly, the hypothesis of an electronic spin state $S=3 / 2$ leads to the best agreement between experiments and calculations.

\section{Conclusions}

We have shown that DANTE spin echoes allow one to measure accurately the distribution of anisotropic hyperfine interactions across severely overlapping NMR signals, as occur in paramagnetic $\mathrm{Fe}(\mathrm{III})$ complexes in solids. This is made possible by the intrinsic excitation properties of DANTE combs of rotor-synchronized pulses: (i) the full inhomogeneously broadened spectrum that spans ca. $600 \mathrm{ppm}$ (ca. $350 \mathrm{kHz}$ ) can be uniformly excited through the use of sub-microsecond pulses, typically $\tau_{p}=0.2 \mu \mathrm{s}$; (ii) a $90^{\circ}$ nutation angle can be achieved for efficient excitation through the cumulative effect of $K$ such pulses applied during $K$ rotor periods (typically $K=5$ ), (iii) a $180^{\circ}$ nutation angle for efficient refocusing results from the cumulative effect of $2 K$ such pulses applied during $2 K$ rotor periods, (iv) the width of the $r f$ spikelets is determined by the overall duration of the spin-echo sequence, which amounts to $3 K$ rotor periods or $230.8 \mu$ s if $v_{\text {rot }}=65 \mathrm{kHz}$ and $K=5$, leading to a width of the $r f$ spikelets of ca. $4 \mathrm{kHz}$. This duration can be optimized to adapt the selectivity if different sites are incompletely resolved. This method can unveil sites that are completely hidden under the inhomogeneous lineshape in conventional spin-echo spectra. The anisotropic pseudocontact shifts of the $\mathrm{Fe}(\mathrm{III})$ complex determined by our method correlate generally well with interatomic distances calculated with DFT methods. This approach may also offer a new means to investigate electronic spin states and thus the electronic configuration of metal centers.
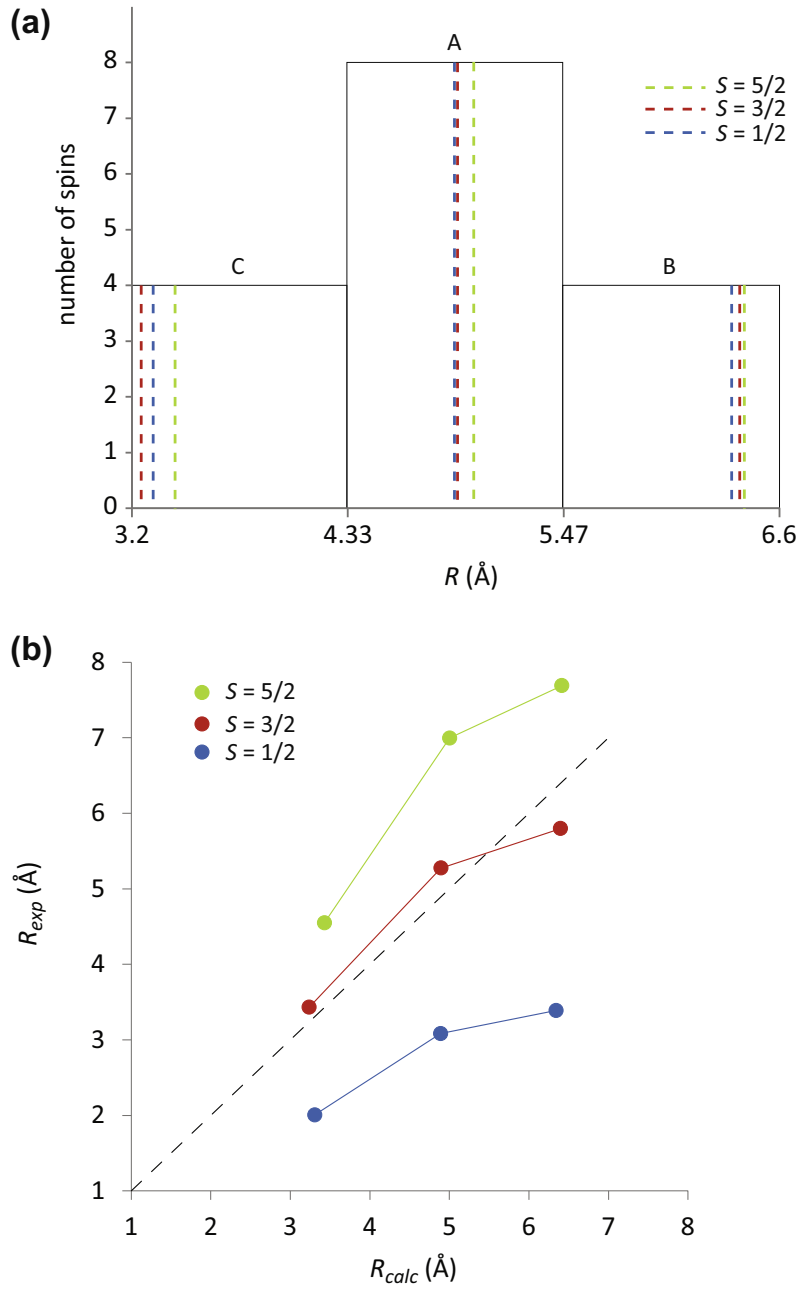

Figure 5. (a) Histogram showing the number of proton spins in a single molecule of the metal complex bis(oxazolinylphenyl)amine- $\mathrm{Fe}(\mathrm{III}) \mathrm{Cl}_{2}$ according to their distance from the paramagnetic Fe(III) center, grouped in three 'bins' of $1.13 \AA$ each, starting from $3.2 \AA$. The vertical dashed lines represent the average distances within each box for the hypotheses $S=1 / 2,3 / 2$ and $5 / 2$, in blue, red and green, respectively. (b) Correlation between $R_{\exp }$ calculated with Eq. (2) from the parameters $\Delta_{\text {aniso }}$ of Table 1 and the average $R_{\text {calc }}$ obtained from the DFT-optimized geometries of Figure $1 \mathrm{~b}$. The color-coding is the same as in (a). The errors in the vertical experimental axis always fall within the size of the dots.

\section{Experimental and computational details}

All ${ }^{1} \mathrm{H}$ MAS spectra have been recorded on a Bruker 800 spectrometer $\left(18.8 \mathrm{~T}, 800.0 \mathrm{MHz}\right.$ for $\left.{ }^{1} \mathrm{H}\right)$ equipped with an Avance-II console, using $1.3 \mathrm{~mm}$ zirconia rotors in a triple-resonance probe, and a spinning frequency $v_{\text {rot }}=65 \mathrm{kHz}$. The proton chemical shifts were referenced to adamantane at $1.8 \mathrm{ppm}$. The $r f$-field amplitude was $v_{1}=250 \mathrm{kHz}$. A recovery delay of $2 \mathrm{~s}$ was used in all cases. Numerical fits were performed with dmfit [36] (Figure 2c,d and 4) or with sola as implemented in Topspin 2.1 (Figure 3a-c). The fitting procedures were allowed to explore a space spanned by the following five parameters: the height of the isotropic peak, the isotropic shift, the anisotropy, the asymmetry, and the line broadening. Where applicable, fits for two sites were achieved by allowing all 10 parameters to vary. The error bars shown in Figure $2 \mathrm{c}$, d were estimated by adding random noise proportional to the actual noise level to the experimental spectra and repeating the fitting procedure on the resulting data sets. The difference $\left|\lambda-\lambda_{\text {noise }}\right|$, where $\lambda=\Delta_{\text {aniso }}$ or , was assumed to be representative of the errors. 
The three structures of the Fe(III) complex were firstly optimized with the semi-empirical PM7 method implemented in MOPAC2012 [31]. A further optimization was performed with Gaussian09 [32] using the DFT B3LYP [37,38] hybrid functional and LANL2DZ [33-35] and 6-31+G(d) basis sets for Fe and all other atoms, respectively.

\section{Acknowledgements}

The authors would like to thank Martial Rey for technical assistance and Dr. Herbert Früchtl, Dr. Daniel Abergel and Dr. Daniel F. Jana for constructive discussions. This work was supported by the Swiss National Science Foundation (SNSF), the Ecole Polytechnique Fédérale de Lausanne (EPFL), the Swiss Commission for Technology and Innovation (CTI), a European Research Council (ERC) starting grant no. 257096 to Prof Xile Hu and the French CNRS.

\section{References}

[1] G. Pintacuda, G. Kervern, Top. Curr. Chem. 335 (2013) 157.

[2] A.W. MacGregor, L.A. O’Dell, R.W. Schurko, J. Magn. Reson. 208 (2011) 103.

[3] G. Kervern, G. Pintacuda, L. Emsley, Chem. Phys. Lett. 435 (2007) 157.

[4] A.J. Pell et al., J. Chem. Phys. 134 (2011) 024117.

[5] T. Jovanovic, A.E. McDermott, J. Am. Chem. Soc. 127 (2005) 13816.

[6] S. Balayssc, I. Bertini, M. Lelli, C. Luchinat, M. Maletta, J. Am. Chem. Soc. 129 (2007) 2218.

[7] S. Balayssc et al., ChemBioChem 8 (2007) 486

[8] S. Balayssc, I. Bertini, A. Bhaumik, M. Lelli, C. Luchinat, Proc. Natl. Acad. Sci. U.S.A. 45 (2008) 17248

[9] G. Kerveern, A. D’Aléo, L. Toupet, O. Maury, L. Emsley, G. Pintacuda, Angew. Chem. Int. Ed. 48 (2009) 3082.

[10] G. Kervern et al., J. Am. Chem. Soc. 128 (2006) 13545.

[11] A.N. Clayton, C.M. Dobson, C.P. Grey, J. Chem. Soc., Chem. Commun. 1 (1990) 72

[12] K. Liu, D. Ryan, K. Nakanishi, A. McDermott, J. Am. Chem. Soc. 117 (1995) 6897.

[13] Y. Ishii, N.P. Wickramasinghe, S. Chimon, J. Am. Chem. Soc. 125 (2003) 3438.
[14] N.P. Wickramasinghe, M. Shaibat, Y. Ishii, J. Am. Chem. Soc. 127 (2005) 5796

[15] G. Bodenhausen, R. Freeman, G.A. Morris, J. Magn. Reson. 23 (1976) 171.

[16] P. Caravatti, G. Bodenhausen, R.R. Ernst, J. Magn. Reson. 55 (1983) 88.

[17] V. Vitzthum, M.A. Caporini, S. Ulzega, G. Bodenhausen, J. Magn. Reson. 212 (2011) 234.

[18] V. Vitzthum et al., J. Magn. Reson. 223 (2012) 228.

[19] D. Carnevale, V. Vitzthum, O. Lafon, J. Trébosc, J.-P. Amoureux, G. Bodenhausen, Chem. Phys. Lett. 553 (2012) 68

[20] S.-F. Lu, D.-M. Du, S.-W. Zhang, J. Xu, Tetrahedron: Asymmetry 15 (2004) 3433.

[21] T. Inagaki, A. Ito, J.-I. Ito, H. Nishiyama, Angew. Chem. 122 (2010) 9574.

[22] T. Inagaki, L.T. Phong, A. Furuta, J.-I. Ito, H. Nishiyama, Chem. Eur. J. 16 (2010) 3090.

[23] S. Antonijevic, S. Wimperis, J. Magn. Reson. 164 (2003) 343.

[24] E.L. Hahn, Phys. Rev. 80 (1950) 580.

[25] H.Y. Carr, E.M. Purcell, Phys. Rev. 94 (1954) 630.

[26] G. Bodenhausen, H. Kogler, R.R. Ernst, J. Magn. Reson. 58 (1984) 370.

[27] P. Hodgkinson, L. Emsley, J. Chem. Phys. 107 (1997) 4808.

[28] A.R. Brough, C.P. Grey, C.M. Dobson, J. Am. Chem. Soc. 115 (1993) 7318.

[29] A. Nayeem, J.P. Yesinowski, J. Chem. Phys. 89 (1998) 4600.

[30] N.P. Wickramasinghe et al., J. Chem. Phys. 128 (2008) 052210.

[31] MOPAC 2012, James J. P. Stewart, Stewart Computational Chemistry, Version 13.073W web: Http://Open MOPAC.

[32] M.J.T. Frisch, G.W. Trucks, H.B. Schlegel, G.E. Scuseria, M.A. Robb, J.R Cheeseman, G. Scalmani, V. Barone, B. Mennucci, G.A. Petersson, H. Nakatsuji, M. Caricato, X. Li, H.P. Hratchian, A.F. Izmaylov, J. Bloino, G. Zheng J.L Sonnenberg, M. Hada, M. Ehara, K. Toyota, R. Fukuda, J. Hasegawa, M. Ishida, T. Nakajima, Y. Honda, O. Kitao, H. Nakai, T. Vreven, J.A. Montgomery Jr., J.E. Peralta, F. Ogliaro, M. Bearpark, J.J. Heyd, E. Brothers, K.N. Kudin, V.N. Staroverov, R. Kobayashi, J. Normand, K. Raghavachari, A. Rendell, J.C. Burant, S.S. Iyengar, J. Tomasi, M. Cossi, N. Rega, J.M. Millam, M. Klene, J.E. Knox, J.B. Cross, V. Bakken, C. Adamo, J. Jaramillo, R. Gomperts, R.E. Stratmann, O. Yazyev, A.J. Austin, R. Cammi, C. Pomelli, J.W. Ochterski, R.L. Martin, K. Morokuma, V.G. Zakrzewski, G.A. Voth, P. Salvador, S. Dannenberg, S. Dapprich, A.D. Daniels, Ö. Farkas, J.B. Foresman, J.V. Ortiz, J. Cioslowski, D.J. FoX, GausSian 09, Revision A.1, Gaussian, Inc., Wallingford, CT, 2009.

[33] P.J. Hay, W.R. Wadt, J. Chem. Phys. 82 (1985) 270.

[34] W.R. Wadt, P.J. Hay, J. Chem. Phys. 82 (1985) 284.

[35] P.J. Hay, W.R. Wadt, J. Chem. Phys. 82 (1985) 299.

[36] D. Massiot et al., Magn. Reson. Chem. 40 (2002) 70.

[37] A.D. Becke, J. Chem. Phys. 98 (1993) 5648.

[38] C. Lee, W. Yang, R.G. Parr, Phys. Rev. B 37 (1988) 785. 